\title{
« Théâtre de narration » en Italie
}

Entre témoignage et affabulation

\section{Élodie Cornez}

\section{OpenEdition}

Journals

Édition électronique

URL : https://journals.openedition.org/cher/349

DOI : $10.4000 /$ cher.349

ISSN : 2803-5992

Éditeur

Presses universitaires de Strasbourg

\section{Édition imprimée}

Date de publication : 19 novembre 2020

Pagination : 103-112

ISBN : 979-10-344-0071-3

ISSN : 1968-035X

Référence électronique

Élodie Cornez, " «Théâtre de narration » en Italie », reCHERches [En ligne], 25 | 2020, mis en ligne le 01 octobre 2021, consulté le 19 novembre 2021. URL : http://journals.openedition.org/cher/349; DOI https://doi.org/10.4000/cher.349 


\title{
"Théâtre de narration » en Italie Entre témoignage et affabulation
}

\author{
ÉlOdie CORnEZ ${ }^{1}$
}

$L^{c}$ e «théâtre de narration» ou "théâtre-récit» («teatro di narrazione»), qui constitue l'une des orientations les plus significatives de la scène italienne contemporaine, naît au début des années quatre-vingt-dix. Il ne s'agit pas à proprement parler d'un mouvement collectif - d'ailleurs aucun des représentants de cette forme n'accepte l'appellation "théâtre-narration", mais plutôt de la convergence, au même moment, de poétiques similaires, même si chacun de ces auteurs-acteurs-narrateurs possède sa propre pratique théâtrale. Cependant, on peut dégager certaines caractéristiques communes: c'est avant tout un théâtre de l'acteur seul sur scène, acteur-narrateur - narracteur (narrattore) selon un néologisme de Pier Giorgio Nosari (2004: 11) - qui est aussi, dans la plupart des cas, le principal auteur de ses textes, même lorsqu'il se fait aider par des collaborateurs; c'est également un théâtre plutôt pauvre: le décor se réduit parfois à une simple chaise installée sur la scène. Enfin, les sujets abordés concernent presque toujours un épisode de l'histoire d'Italie ou un aspect de la société contemporaine - c'est pourquoi l'expression «teatro civile» est aussi parfois employée pour le désigner.

La question qui se pose naturellement pour ce genre de théâtre est donc la suivante: comment le genre dramatique, qui est par essence représentation, adaptation, voire illusion, se confronte-t-il au matériel brut que représente un événement clé de l'histoire ou de la chronique italienne? Que font ces acteursnarrateurs ou narracteurs: se contentent-ils d'évoquer ces événements? De témoigner? À moins qu'ils n'affabulent?

Chacun de ces termes a son importance, nous en examinerons les enjeux dans un contexte dramaturgique qui, après les mouvements néo-avant-gardistes des années soixante et soixante-dix, se fonde de nouveau sur la parole, dont il conviendra d'interroger le caractère «véridique».

1 Professeure agrégée, docteure, PRAG à l'Université de Lille 3. 
Le corpus sur lequel s'appuie cette étude est constitué de trois spectacles créés par trois acteurs différents: Marco Baliani, Marco Paolini et Ascanio Celestini.

Marco Baliani, né à Rome en 1950, est considéré comme le créateur du premier spectacle de narration en 1990, intitulé Kohlhaas. En 1998, il crée Corpo di Stato, il Delitto Moro, spectacle évoquant les cinquante-cinq jours de détention, puis l'assassinat, d'Aldo Moro, homme politique de la Démocratie Chrétienne dans la Rome de la fin des années soixante-dix où règne un climat extrêmement tendu, qu'a connu le jeune Baliani, alors étudiant en architecture, l'une des facultés parmi les plus engagées.

Marco Paolini (1956), de six ans son cadet, se fait essentiellement connaître grâce à la retransmission télévisée d'un de ses premiers spectacles, Vajont, 9 ottobre 1963. Il y raconte la catastrophe du barrage du Vajont, le lac de retenue artificiel le plus important des Alpes, à la frontière entre le Frioul et la Vénétie, construit dans un contexte de frénésie industrielle à l'époque où l'Italie, en pleine expansion économique, cherche à valoriser une des rares ressources énergétiques de son territoire: l'eau. Le terrain géologique montagneux finit par céder sous la pression de l'eau, un pan de la montagne s'écroule dans le lac, et une vague gigantesque passe par-dessus le barrage et déferle sur la vallée, faisant plus de 5000 morts, notamment dans la petite ville de Longarone. La retransmission du spectacle - d'une durée de trois heures - en direct sur la RAI depuis le site du Vajont, réunit, en octobre 1997, plus de trois millions de téléspectateurs.

Ascanio Celestini (1972), plus jeune que les deux précédents, crée, en 2000, Radio Clandestina. Memoria delle Fosse ardeatine. Il y rapporte le massacre, en 1944, de plus de trois cents civils, en représailles à une embuscade tendue aux nazis par les partisans dans une rue de Rome.

On lit souvent dans les comptes rendus des spectacles relevant de la «narration civile» des commentaires comme: "le narrateur témoigne de...», «un témoignage très fort sur cette période de notre histoire...». Le narrateur ou plutôt le «narracteur» - narrattore - serait donc un témoin ou serait du moins investi de cette fonction de témoin. Il nous faut d'abord revenir à la définition ce qu'on entend par "témoin» et "témoignage». Le témoin est la personne qui a été présente au moment d'un fait et «qui certifie ou qui peut certifier ce qu'elle a vu ou entendu $»^{2}$. Ce témoin raconte un fait auquel il a assisté et le témoignage est le récit qu'il en fait, utile pour tenter de reconstruire l'événement tel qu'il s'est produit. En réunissant plusieurs témoignages, on multiplie les points de vue sur un même événement et l'on peut ainsi tendre vers une plus grande précision et vérité des faits.

Le témoin est donc présent au moment des faits, c'est sa caractéristique première, même s'il n'est pas directement impliqué dans l'événement. De son témoignage, il est exigé qu'il dise la vérité, en particulier quand il témoigne dans

2 Définition du Petit Robert. 
un cadre juridique, sous serment. Il se doit de relater ces faits avec le plus de précision et d'objectivité possibles.

Cette définition sommaire ne semble en rien correspondre à celle du narrateur théâtral en position de témoin. Dans un spectacle de narration, exception faite, peut-être, dans une création comme Vajont, la précision n'est pas la priorité, pas plus que l'objectivité, ni même la vraisemblance: les récits de guerre d'A. Celestini, par exemple, virent parfois au fabuleux avec l'introduction d'éléments clairement invraisemblables. Le narrateur semble donc se trouver davantage du côté de l'affabulation, dans le sens qu'en donne un dictionnaire comme le Petit Robert, qui indique qu'affabuler consiste à organiser en épisodes continus le thème d'une œuvre d'imagination, à raconter, à présenter sous forme d'une fable, à arranger la réalité à sa manière. Voilà qui jette le soupçon sur la véracité et fiabilité du récit - de l'affabulation - du narrateur - narracteur.

Le récit du témoin - deuxième caractéristique - est rigoureusement chronologique, et s'il ne l'est pas, les questions du magistrat y mettront bon ordre. Le récit du narrateur est cyclique et volontairement répétitif. Le narrateur, qui a le souci de capt(iv)er son auditoire, peut émailler son récit de la même phrase ou du même thème, en des endroits stratégiques, manière de le structurer pour guider le public et de susciter son attention.

C'est que le récit du narrateur-narracteur n'est pas destiné à instruire une procédure juridique, il relève de l'art et jouit par conséquent de la plus grande liberté... d'affabulation. Il s'adresse à un public d'auditeurs, il a été travaillé, organisé, contrairement au témoignage, en principe immuable, qui se doit d'être reproduction spontanée, non retravaillée, fidèle - à chaque fois qu'il est répété - de ce à quoi le témoin direct a assisté. En revanche, le narracteur n'est tout au plus qu'un témoin de second degré, un témoin - au sens de relai - de témoignages, «un témoignage de témoignage» comme l'expose A. Celestini: «Moi je suis témoin d'un témoignage et l'événement dont j'ai été témoin est le récit.» (Celestini 2007: 135) ${ }^{3}$.

Pour préparer leur spectacle, ces acteurs-narrateurs réalisent un travail de documentation conséquent, parfois digne d'un historien. Ils lisent, font des recherches dans les archives, interrogent des témoins directs, les acteurs des événements. Leur connaissance du sujet est donc a priori indirecte. Mais A. Celestini, s'amuse à brouiller la notion même de témoignage et de témoin, se posant comme «le témoin du récit du témoin». En démultipliant les niveaux de

3 «Io racconto la mia testimonianza, che è quasi sempre la testimonianza di un'altra testimonianza. Anzi in realtà non racconto mai il mio essere testimone dell'avvenimento: io sono testimone di una testimonianza e l'avvenimento di cui sono stato testimone è il racconto.» (Celestini 2007: 135).

«Moi je raconte mon témoignage, qui est presque toujours témoignage d'un autre témoignage. Et même, en fait je ne raconte jamais que j'ai été témoin de l'événement: moi je suis témoin d'un témoignage et l'événement dont j'ai été témoin est le récit». La traduction est de nous. 
témoignages et en jouant avec la notion de récit, il jette le doute sur la fiabilité même du témoignage et réinterroge la figure même du témoin.

Si nous revenons à la définition sommaire que nous avons en donnée, il est celui qui, ayant été présent au moment de l'événement, en est revenu pour le raconter. Souvent, il n'en a pas été l'acteur, mais seulement le spectateur. Cependant, passif au moment $\mathrm{x}$, il devient actif au moment $\mathrm{x}+1$, lorsqu'il se met à raconter ce dont il a été témoin. Le témoignage est d'abord un récit, et la narration un acte qui suppose un sujet actif, et donc une subjectivité. Le simple fait de raconter contraint le témoin, dont on attend la plus grande objectivité, à replacer les événements dans un cadre temporel, dans une certaine cohérence logique qui peut en dénaturer l'exactitude. L'ambiguïté du témoin provient aussi de sa position indéfinie: il n'est ni impliqué dans les faits, ni complètement extérieur à ceux-ci - surtout si ce qu'il a vu lui a causé un traumatisme, par exemple. Le témoin est quelqu'un que le hasard a placé là, sans qu'il ait nécessairement les outils logiques, scientifiques, historiques, sociologiques pour rendre compte des faits. Il les restitue avec ses moyens et ses mots, nécessairement empreints de subjectivité. Il ne peut qu'offrir un témoignage approximatif, biaisé. Par ailleurs, le témoignage, dans la plupart des cas, se fait oralement, et le témoin raconte, convertit en mots les actions parfois très rapides qu'il a vues, mais aussi ses propres impressions et sensations. Avant que le récit ne soit fixé définitivement par l'écrit - par un procès-verbal, par un livre - le témoin aura réitéré plusieurs fois son récit pour ses proches, pour les historiens, pour les enquêteurs. Le récit ne pourra que se modifier d'une narration à l'autre, les fluctuations de la mémoire feront que les détails se préciseront ou s'effaceront, et l'imagination viendra combler, étoffer, voire modifier le récit. Finalement, les frontières entre narration et témoignage se révèlent plus floues et plus ambiguës qu'il n'y paraît.

Le narracteur se pose donc comme «témoin » dans ce «théâtre de narration" alors que la plupart du temps, comme l'a signalé A. Celestini, il n'a qu'une connaissance tout à fait indirecte des faits dont il se dit le témoin.

Dans les trois spectacles de notre corpus, le narracteur n'a jamais assisté en personne aux faits appartenant à la mémoire collective qu'il évoque. M. Paolini n'était pas à Longarone lorsque la vague a submergé le barrage; M. Baliani n'a pas participé à l'enlèvement d'Aldo Moro; et A. Celestini n'a pas connu le massacre des Fosse ardeatine. Cependant, chacun d'eux entretient des liens particuliers avec ces événements respectifs. Ce sont des événements qui les ont marqués personnellement d'une façon ou d'une autre. A. Celestini écoutait les récits de guerre de son père et de son grand-père. $M$. Paolini habitait alors à Belluno, non loin du barrage, et la gare de Longarone faisait partie du chemin des vacances. Il a gardé le souvenir de sa mère pleurant à côté du poste de radio, au moment où la nouvelle de la catastrophe a été diffusée sur les ondes. Tous ont été témoins de l'époque ou du lieu évoqués dans leurs récits : le climat politique exacerbé dans la capitale en 1978 chez M. Baliani; la ville de Rome et la ruelle de l'attentat pour A. Celestini. C'est à partir de ce point d'ancrage spatiotemporel que commence le travail de reconstitution des faits en entremêlant le 
témoignage de leur propre expérience et le témoignage de l'expérience collective. L'une des notions-clé du théâtre de narration est justement «l'expérience» qui vient apporter une valeur ajoutée au témoignage du narracteur. L'expérience est l'apport personnel, le support qui lui appartient en propre et sur lequel il va bâtir sa narration. Comme le souligne Marco Baliani: "Dans le corps à corps du théâtre, récit et autobiographie, fable et réalité, mythe et histoire personnelle s'entremêlent dans l'idiome commun de la narration ${ }^{4}$. " Cette analyse du metteur en scène Gabriele Vacis, qui a beaucoup travaillé avec les acteurs-narrateurs, est particulièrement éclairante à ce propos

La mémoire produit des images et des histoires, des histoires et des images qui sont à la base de tout processus de communication. Chaque acteur doit construire son propre théâtre de la mémoire. Chaque acteur doit mettre en relation les grands faits de l'histoire et de l'époque avec ses propres faits, ses propres expériences. Ce n'est qu'ainsi qu'il peut trouver cette urgence, cette nécessité dont il a besoin pour être sur scène, ce n'est qu'ainsi qu'il peut se déclarer auteur de sa présence sur scène. (Vacis 2005: 112)

L'affaire Moro entre en parfaite résonnance avec l'expérience personnelle de Marco Baliani, alors actif dans les mouvements d'extrême-gauche à la fin des années soixante-dix: M. Baliani raconte le climat de suspicion qui s'installe à Rome après l'enlèvement, les barrages policiers, les fouilles, la peur de revoir les amis qui ont franchi le pas de la lutte armée, et le doute, omniprésent, sur le sens de cette action politique. Il ne s'agit pas d'une reconstitution, point par point, des cinquante-cinq jours de détention - les précisions historiques n'apparaissent que rarement dans le récit, et seulement en toile de fond. Le temps est davantage scandé par l'évolution des réflexions du narracteur, d'abord vaguement enthousiaste, puis de plus en plus incertain, et enfin horrifié par une atmosphère de plus en plus lourde, par ses discussions avec les compagni, dont certains choisirent à ce moment la clandestinité. Ainsi que le dit Walter Benjamin, la narration ne consiste pas à transmettre l'événement pour luimême, comme le ferait une information ou un compte rendu, mais l'intègre à la vie du narrateur. Le récit porte nécessairement sa marque (Benjamin 2006: 256). On a donc une subjectivité nécessaire dans le récit de narration, et même assumée: le narrateur ne peut ressentir cette urgence de raconter si les faits n'ont pas d'abord été filtrés à travers lui, si la mémoire historique et sa mémoire personnelle ne s'entremêlent pas. Le récit possède une ampleur, une vibration

4 «Nel corpo a corpo del teatro, racconto e autobiografia, favola e realtà, mito e storia personale si intrecciano nell'idioma comune della narrazione», Marco Baliani cité dans (Fiaschini et Ghiglione 1998: 8).

5 [...] la memoria produce immagini e storie, storie e immagini che sono alla base di qualsiasi percorso comunicativo. Ogni attore deve costruirsi il proprio teatro della memoria. Ogni attore deve mettere in relazione i grandi fatti storici ed epocali con i propri fatti, le proprie esperienze. Solo così può trovare quell'urgenza, quella necessità che gli occorre per stare in scena, solo così può dirsi autore del suo stare in scena. (Vacis 2005: 112). 
qui manque à la simple information. L'acteur-narrateur n'est ni un historien, ni un journaliste, il n'a donc pas d'obligation par rapport à une vérité unique qui serait celle des faits. La notion même de vérité prend une tout autre valeur dans la narration: pour Gerardo Guccini, qui a mené une étude très intéressante sur le «théâtre de narration", il ne s'agit pas pour le narrateur de "témoigner du vrai», mais de "produire des témoignages vrais», qui attestent que le contenu de la matière narrée est enraciné dans la personne même du narrateur (Guccini 2005: 13), qu'il fait donc écho à une vérité intérieure. Marco Baliani va même jusqu'à établir que «La vérité n'existe pas comme objet de savoir mais comme condition de l'expérience ${ }^{6} . »$

Et qu'en est-il du côté de la réception? Les spectateurs sont pris entre affabulation et témoignage et leur participation active est essentielle pour que le spectacle trouve tout son sens. Le spectateur sait qu'il vient voir un «spectacle sur» mais il peut éprouver un certain malaise face à une fiction, celle du théâtre, qui ne rend pas compte des événements avérés et connus de tous, conformément à la représentation que tout le monde en a. Les faits, plus évoqués qu'expliqués, sont en outre truffés d'inventions narratives ou de digressions autobiographiques de l'acteur. L'objectif est bien d'amener les spectateurs à s'interroger sur leurs souvenirs personnels: comment ont-ils vécu la période de l'événement, l'événement lui-même, le moment de son annonce? C'est bien à l'expérience personnelle de chacun des spectateurs que s'adresse le récit de l'acteur-narrateur, qui entre alors en résonnance avec elle, convoque la mémoire personnelle et les émotions de chacun. Il s'agit de donner toute son ampleur à la rencontre des expériences, bien au-delà de la simple nouvelle de journal télévisé.

Le récit se veut ouvert sur les possibles et n'impose pas de point de vue arrêté, du moins en théorie: le but n'est pas de désigner les coupables, mais bien de parcourir les faits, de les faire revivre le temps d'une représentation pour mieux les connaître et les comprendre. La «liberté»du spectateur n'est cependant pas illimitée, celui-ci suit le cheminement mental de l'acteur-narrateur; il est nécessairement influencé par la présentation des faits qui lui est proposée qui n'est pas neutre - ne peut pas être neutre. Les convictions politiques personnelles de chacun des trois acteurs sont par ailleurs parfaitement connues des spectateurs. La pièce propose une narration-expérience: aux spectateurs de mener leur propre réflexion. Comme le dit Baliani citant Pasolini, l'art de la narration, lorsqu'il est poussé à son point le plus haut, permet à la pièce d'ouvrir non pas sur un message unique mais de susciter des vérités multiples, car les spectateurs qui écoutent - avec leur propre expérience - sont tous différents ${ }^{7}$.

6 «La verità non esiste come oggetto di sapere ma come condizione dell'esperienza », Marco Baliani cité dans (Ghiglione 1998: 34).

7 «Pasolini dice che quando l'artificio, l'arte, la tecnica arrivano ad un punto di abilità così alta, diventano sacre. La tecnica del raccontare va affinata al punto che non ci si accorga più che c'è : in quei momenti il racconto veicola una verità. Il messaggio non è unico, arrivano più verità, perché le persone che ascoltano sono diverse.» (Baliani 2005: 60). 
Certains narracteurs invitent très explicitement les spectateurs à garder une distance critique. Marco Paolini, par exemple, interrompt son spectacle pour mettre en garde son public: "Comment puis-je vous dire tout cela? Ça fait quarante minutes que vous m'écoutez, vous avez bien dû avoir parfois des doutes sur ce que je raconte! Je ne suis pas technicien, je ne suis pas avocat, je ne suis pas diplômé, je suis acteur! [...] si aucun doute ne vous a assaillis, ce n'est pas bien ${ }^{8}$ » Le concept même de vérité brute n'entre pas en ligne de compte dans un travail théâtral, même extrêmement documenté et consciencieux: l'art de raconter des histoires consiste à trouver les moyens de maintenir l'attention pendant les deux à trois heures que dure le spectacle. L'objectif est de rendre digestes les passages particulièrement techniques ou complexes: "Alors mettons-nous d'accord: pour vous tenir éveillés, $j^{\prime}$ invente quelquefois ${ }^{9}$ ", précise le narracteur dans Vajont. Chez Ascanio Celestini, le récit glisse régulièrement vers un registre fictionnel, voire suréel parfaitement assumé, ce qui remobilise l'attention du public.

L'une des règles à respecter est celle de l'identité de l'acteur, quidoit être parfaitement claire. Il s'agit bien pour le narracteur de s'exposer à la première personne et non pas derrière le masque d'un personnage, ni être une simple voix neutre qui serait pure énumération de dates et de faits. Cependant, il faut de même éviter l'écueil inverse: l'acteur, maître sur la scène, peut exercer une fascination facilement exploitable. Si l'acteur est doué, dit Marco Paolini, et si de surcroît il ponctue ses affirmations de noms, de données et de dates, ce qu'il dit apparaîtra d'abord vraisemblable puis, au fur et à mesure, vrai. Or, il importe que l'acteur, comme le spectateur, sans cesse remette en question cette capacité de persuasion: "À la fin je dis toujours: "vous avez le droit et même le devoir de douter de ce que vous avez entendu”. ${ }^{10}$ »

«Pasolini dit que lorsque l'artifice, l'art, la technique arrivent à un si haut niveau d'habileté, ils deviennent sacrés. La technique du récit doit être affinée jusqu'à ce que l'on ne s'aperçoive plus qu'elle existe: dans ces moments-là le récit véhicule une vérité. Le message n'est pas unique, ce sont plusieurs vérités qui parviennent, car les personnes qui écoutent sont toutes différentes»

8 «Come faccio a dirvi queste cose? Sono quaranta minuti che mi state ascoltando, vi verranno dei dubbi ogni tanto su quello che sto dicendo! Non sono un tecnico, non sono un avvocato, non sono laureato, sono un attore! [...] se non vi sono venuti dubbi, male!» (Paolini 1999).

9 «Allora ci mettiamo d'accordo: io per tenervi svegli ogni tanto invento.» (Paolini 1999).

10 «Alla fine del racconto dico sempre: "Avete il diritto e anche il dovere di dubitare di tutto quello che avete ascoltato".» (Paolini, Ponte di Pino 1999: 53). La citation continue ainsi : «La sera del 9 ottobre 1997, alla fine del racconto, con tutto il pubblico in piedi e in silenzio, ero così emozionato che me ne sono dimenticato, e adesso mi rode.» («Le soir du 9 octobre 1997, à la fin du récit, à cause de tout le public debout et silencieux, j'étais tellement ému que j'ai oublié de le dire, et maintenant cela me ronge»). 
Le critère pour juger de la performance du narracteur ne repose donc pas sur la vérité de ce qui est dit, même si l'acteur a choisi de construire son discours sur «le fait de savoir qu'il ne ment pas» (Paolini/Ponte di Pino 1999: 53):

[...] parler de témoignage, de légitimité du témoignage, signifie que les spectateurs s'attendent à ce que vous disiez une chose vraie. Malheureusement, ce consensus est souvent considéré comme une sorte de chèque en blanc. La télévision se sert de ce mécanisme, l'amplifie. La terrible responsabilité morale de ceux qui font ce travail est de dire "J’ai parlé juste”. Moi je peux seulement dire “j'ai parlé honnêtement”. (Paolini/Ponte di Pino 1999: 48) $)^{11}$

Même dans le spectacle du Vajont, qui est pourtant parsemé de chiffres, de noms, de détails techniques assez précis, Marco Paolini ne cesse jamais de témoigner en tant que Marco Paolini, c'est-à-dire en tant qu'individu restituant ce qu'il a compris, lu, appris sur la catastrophe. Jamais le spectacle ne franchit le pas de dire: ceci est une reconstitution scientifique et fiable des faits. La narration ne se construit pas sur un seul plan, elle est au contraire constituée d'un ensemble de strates qui en font toute sa complexité. G. Guccini l'a parfaitement compris lorsqu'il dit que la narration est aussi toujours témoignage d'elle-même, c'est-àdire de la façon dont les faits se sont sédimentés, agencés, reconstitués dans la mémoire du narrateur:

[Le narrateur] exerce instinctivement deux types de témoignage: le témoignage de ce que lui-même a vécu en travaillant à la narration, et celle des référents extérieurs - faits, livres, personnes - qui se sont ainsi imprimés dans les replis de son existence en suscitant l'acte psychique du récit. (Guccini 2005: 7) ${ }^{12}$.

Cette double nature constitue justement la marge d'autocritique que contiennent les spectacles de narration, où le narracteur est toujours conscient de qui il est et de ce qu'il est en train de faire. Il faut à tout moment "opérer une remise à distance» («riaggiustare le distanze», Baliani 2001: 94), ainsi que le préconisait la distanciation brechtienne.

En conclusion, on peut dire que dans le "teatro civile" - "théâtre-récit" ou "théâtre de narration" les faits qui font l'objet du spectacle sont évoqués selon les modalités à la fois du témoignage (au moins de second degré, témoignage de témoignage) et de l'affabulation, qui ne cessent de se recouper et de mêler leurs frontières aux contours poreux. En racontant certains épisodes de l'histoire de l'Italie, c'est en partie d'eux-mêmes que les acteurs-narrateurs témoignent et seul

11 «[...] parlare di testimonianza, di legittimità della testimonianza, significa che gli spettatori si aspettano che tu dica una cosa vera. Purtroppo di solito questo imprimatur viene considerato quasi come una delega in bianco. La televisione avvala questo meccanismo, lo potenzia. La responsabilità morale tremenda di chi fa questo lavoro è di dire "L'ho detta giusta". Io posso solo dire "L'ho detta onestamente" " (Paolini, Ponte di Pino 1999: 48).

12 «[Il narratore] esercita istintivamente due tipi di testimonianza: la testimonianza di quanto egli stesso ha esperito lavorando alla narrazione, e quella dei referenti esterni - fatti, libri, persone - che si sono così incuneati fra le pieghe del suo vivere suscitando l'atto psichico del racconto» (Guccini 2005: 7). 
ce lien organique avec le récit leur confère l'autorité légitime pour le transmettre. Finalement, l'acteur-narrateur est un peu comme le Prologue du théâtre antique: membre de la communauté des citoyens, sans qualification particulière, il se détache de cette même communauté - le chœur - en s'avançant sur scène, mais pour s'adresser à la communauté réelle, celle des spectateurs, afin de leur raconter - et non de leur jouer en créant l'illusion - le drame. Le spectateur est alors appelé à faire son propre chemin, à mêler ses propres expériences à cette mémoire qui vient de lui être rappelée, non pas selon des modalités entièrement et rigoureusement rationnelles et scientifiques, mais aussi et surtout à travers une narration qui offre une dimension supplémentaire au traitement de l'information: la dimension poétique.

\section{Bibliographie}

Baliani M., 2001, «Narrazione e ascolto. Incontro con Marco Baliani», dans Vicenzo Maria Oreggia, Archivio di voci: incontri con Marco Baliani, Gabriella Bartolomei, Moni Ovadia, Marco Paolini, Luca Ronconi e Giuliano Scabia, Milano, Archinto.

Baliani M., 2005, «Esperienza-tempo-verità: seminario sulla narrazione», dans Gerardo Guccini, La bottega dei narratori: storie, laboratori e metodi di Marco Baliani, Ascanio Celestini, Laura Curino, Marco Paolini, Gabriele Vacis, Roma, Audino.

Benjamin W., 2006, «Il narratore. Considerazioni sull'opera di Nicola Leskov» dans Walter Benjamin, Angelus Novus, Torino, Einaudi.

Bologna P., 2007, Tuttestorie. Radici, pensieri e opere di Ascanio Celestini, Milano, Ubulibri.

Fiaschini F., Ghiglione A., 1998, Marco Baliani: racconti a teatro, Firenze, Loggia de' Lanzi.

Ghiglione A., 1998, "A proposito del concetto di duratura», dans Fabrizio Fiaschini et Alessandra Ghiglione, Marco Baliani: racconti a teatro, Firenze, Loggia de' Lanzi.

Guccini G., 2005, La bottega dei narratori: storie, laboratori e metodi di Marco Baliani, Ascanio Celestini, Laura Curino, Marco Paolini, Gabriele Vacis, Roma, Audino.

Nosari Pier G., 2004, «I sentieri dei raccontatori di storie: ipotesi per una mappa del teatro di narrazione", Prove di Drammaturgia. Per una nuova performance epica, 1, luglio 2004, anno X, p. 11-14.

Paolini M., Ponte di Pino O., 1999, Quaderno del Vajont, Torino, Einaudi.

Paolini M., Ponte di Pino O., 1999, Vajont. 9 ottobre 1963. Orazione civile, [vidéocassette] composition et mise en scène de Gabriele Vacis et Marco Paolini, Torino, Einaudi. 
Vacis G., 2005, «Il teatro è narrazione», dans Gerardo Guccini, La bottega dei narratori: storie, laboratori e metodi di Marco Baliani, Ascanio Celestini, Laura Curino, Marco Paolini, Gabriele Vacis, Roma, Audino. 